\title{
A New Paradigm for Personalized Medicine and Companion Diagnostics: The Contract Diagnostics Organization
}

\author{
Philip D. Cotter*,1, Deepti Babu ${ }^{2}$ and Mathew W. Moore ${ }^{1}$ \\ ${ }^{1}$ Research Dx, Irvine, California, USA \\ ${ }^{2}$ University of Alberta, Department of Medical Genetics, Edmonton, Alberta, Canada
}

\begin{abstract}
In the era of personalized medicine, pharmaceutical companies are actively seeking partners to develop Companion diagnostics. Common choices of partners include large diagnostic manufacturers and traditional Contract Research Organizations (CROs), neither of which provides an entire solution. Diagnostics companies have an intrinsic bias toward internal product lines, and are therefore incentivized to direct diagnostics development toward an existing technology platform. The expertise of a CRO lies in the monitoring of pharmaceutical programs, not in diagnostics. In addition, few traditional partners have significant clinical laboratory experience or offer accredited clinical laboratories to ensure that the diagnostic clinical trials are designed for, and conducted in, laboratories that meet regulatory standards. Lastly, these organizations are inexperienced in managing and coordinating the multiple partners required in the development process.

The Contract Diagnostics Organization (CDO) is a new concept designed to aid pharmaceutical companies in addressing challenges in companion diagnostics development. This business model provides pharmaceutical companies a complete outsourcing partner to initiate and manage the parallel development of companion diagnostic tests in synergy with drug development. The CDO combines all of the necessary services, including diagnostics research, an accredited clinical laboratory, project management and regulatory, manufacturing, and consulting in an integrated, technology-independent manner. Thus, the CDO focuses on its pharmaceutical partner's business objectives and ensures the speediest path to market a valuable, personalized drug for patients.
\end{abstract}

Keywords: Personalized Medicine, Companion diagnostic, Contract Research Organization (Cro), Contract Diagnostic Organization (Cdo).

\section{INTRODUCTION}

For many years now, the public, scientific, and business communities have been anticipating the era of personalized medicine. As the concept has progressed and regulatory input has increased with it, the need for high-quality and highly efficient companion diagnostic development has also grown. In response, pharmaceutical companies have actively sought appropriate partners to develop companion diagnostics.

Common choices for partners to date have been large diagnostics companies and traditional contract diagnostics organizations (CROs). Traditional contract diagnostics companies have their own product lines and may therefore not choose approaches that best target the pharmaceutical companies' objectives. CROs also may not have the specific expertise necessary to develop diagnostics, as they have the most experience with pharmaceuticals. In addition, few traditional partners can ensure that clinical trials for diagnostics are conducted in accredited clinical laboratories that meet the clinical regulatory standards, as opposed to the self-regulated good clinical practice and good laboratory practice standards that are applied to clinical research studies. Lastly,

*Address correspondence to this author at the ResearchDx, 13766 Alton Parkway Suite 147, Irvine, CA 92618, USA; Tel: 949-812-6902;

Fax: 949-297-3983; E-mail: pcotter@researchdx.com there are numerous overall logistical complexities that arise when managing and coordinating multiple partners in the drug development process.

Here, we discuss the Contract Diagnostics Organization (CDO), a new concept designed to assist pharmaceutical companies and address challenges in companion diagnostics development. This business model allows pharmaceutical companies a start-to-finish partner to initiate the parallel development of companion diagnostic tests in synergy with drug development.

\section{THE PROMISE OF PERSONALIZED MEDICINE}

"Personalized medicine" refers to the customization of medical treatment to the individual characteristics of each patient [1]. The term "personalized medicine" was coined in the 1990s, although the premise predated this [2].The advantages to a personalized medicine approach are theoretically clear; personalized medicine has the power to more efficiently, effectively, and safely direct health care than traditional non-targeted approaches.

Recently, the interest in personalized medicine has increased substantially; based on PubMed searches on the term 'personalized medicine' a 2011 publication found that the number of scientific publications on the subject has shown an exponential growth in the period from 1999 to 2010 [3]. From the financial perspective, the U.S. personalized medi- 
cine market was estimated to be $\$ 232$ billion in 2009 [4]. It is projected to grow eleven percent annually and nearly double in size to over $\$ 450$ billion by 2015. The core diagnostic and therapeutic segment of the market - comprised primarily of pharmaceutical, medical device and diagnostics companies - was estimated at $\$ 24$ billion and expected to grow by 10 percent annually, reaching $\$ 42$ billion by 2015 [4].

On average, a traditional therapeutic works effectively for about $50 \%$ of individuals [5], with hypersensitivity, lack of response, inability to metabolize, and adverse reactions being observed in the remainder of patients. In a recent report, it was estimated that more than $5 \%$ of hospital admissions were associated with adverse reactions to prescribed drugs [6]. Many of these are due to individual genetic differences that render one hypersensitive to the drug, or unable to metabolize it properly [7]. One intent of a personalized medicine approach is to reduce these adverse events substantially and increase the effectiveness of treatment; the right drug at the right dose, for the right individual at the right time.

\section{COMPANION DIAGNOSTICS IN PERSONALIZED MEDICINE}

The commonly applied therapeutic model of "one drug for one disease" does not fit heterogeneous disease mechanisms at the molecular level [3]. A stratified approach, identifying groups of patients based on certain biologic characteristics or biomarkers has the potential to be more efficient and effective while reducing undesirable drug interactions and side-effects [3]. Even today, stratification is a key component of both diagnosis and therapy selection. Drug and dosages are modified according to key markers including patient weight, ethnicity, sex, age, and traditional diagnostics such as blood and urine analyses.

Companion diagnostics extendthis concept to include diagnostic biomarker assays and genetic testing, the results of which can be used in several modalities. A companion diagnostic result may be used to stratify patients to identify those that will respond to a particular therapeutic. Similarly, it can identify those patients that will not respond, allowing health care providers to more rapidly move onto a treatment that may be effective. For example, detection ofHER2amplification by molecular cytogenetics analysis is used to identify patients more likely to respond totrastuzumab (Herceptin $^{\circledR}$ ) in breast and gastric cancers [8].

A second important use of companion diagnostics is in customizing drug dosage based on metabolism biomarker status. For example, warfarin dosage can be calculated using an algorithm (www.warfarindosing.org) that incorporates a patient's CYP2C9 and VKORC1 genotypes [9]. Individuals with $C Y P 2 C 9 * 2$ and $C Y P 2 C 9 * 3$ genotypes produce an enzyme with reduced capacity (by $30 \%$ and $80 \%$, respecttively) to metabolize warfarin. Those with the 1639G $>$ A VKORC1 genotype produce an enzyme with about 50\% reduced capacity. Traditional dosing leads to delayed and elevated warfarin steady-state levels, and potentially to an increased bleeding risk [10]. As such, reduced warfarin dosages are needed for those with these genotypes [10].

Lastly, a companion diagnostic may be used to monitor treatment. Quantitative analysis of the $B C R-A B L$ fusion tran- scriptsis used to monitor imatinibmesylate (Gleevec ${ }^{\circledR}$ ) treatment in patients with chronic myeloid leukemia [11], and the $P M L / R A R A$ translocation is diagnostic and used to monitor retinoic acid therapy in acute promyelocytic leukemia [11, $12]$.

Companion diagnostics are required to ensure the effective, safe development and use of many personalized therapeutics. The use of genetic, molecular or other companion diagnostics stratifies patient populations by allowing healthcare providers to select a targeted therapeutic based on their patient's particular profile.

The use of composite biomarker signatures, the first step in companion diagnostic development, has become more common in the clinical development of therapeutics. Biomarker research has been of considerable interest to the clinical and basic science communities. For example, between 1986 and 2009, the National Institutes of Health (NIH) awarded over 28,000 grants containing the term "biomarker," [13] a large increase from previous years. The total funding for these awards in 2008 and 2009 alone was over $\$ 2.5$ billion. This demonstrates a notable upward trend in the desire to use biomarkers and personalized medicine to develop clinical therapeutics. As evidence of growth in the industry, $10 \%$ of currently marketed pharmaceuticals include biomarkers on their labels [14].

As an example, patients with metastatic colorectal cancer and somatic mutations in the KRAS gene are unlikely to respond to chemotherapeutic treatment with Erbitux ${ }^{\circledR}$ (cetuximab) and/or Vectibix ${ }^{\circledR}$ (panitumumab) [15].While the results of these studies are well known, and this knowledge is being applied on an ad hoc basis by various clinical institutions, there are currently no approved clinical guidelines or mandated testing for KRAS mutations prior to therapy. Various challenges in the process may contribute to the lack of these guidelines in the U.S. as clearly, an available companion diagnostic (in this case, genetic testing for somatic KRAS mutations in the colorectal tumor) would help physicians guide and refine their treatment options. Not only do companion diagnostics increase drug effectiveness, they can also reduce the likelihood of adverse events, save time, and decrease the financial burden on the healthcare system.

Companion diagnostics are critical to the success of personalized medicine and yet despite this, only a minority (about 1\%) of marketed therapeutics have a companion diagnostic [14]. Responses from the larger pharmaceutical and biotechnology companies to create personalized therapeutics have been lower than expected. Success rates in bringing these drugs to market have also been low. A number of scientific, strategic, commercial, and regulatory factors have been attributed to this [16]. For example, retrospective identification of a useful biomarker presents many technical and regulatory challenges.

\section{REGULATORY AGENCIES WEIGH IN ON COMPANION DIAGNOSTICS}

The emergence of personalized medicine and companion diagnostics to inform clinical decision-making, along with assays to guide drug selection and dose, has led regulatory agencies to respond. The U.S. Food and Drug Administration (FDA), Health Canada and the European Medicines Agency 
(EMA) have either mandated or recommended in several cases that biomarker testing be performed prior to prescribing certain drugs [17]. Specifically, the EMA has acknowledged the relevant biomarker in some companion diagnostic patient information and package leaflets.

In draft guidance from July 2011, the FDA indicated that, "in most circumstances, if use of an in vitro companion diagnostic device (IVD companion diagnostic device) is essential for the safe and effective use of a therapeutic product, [it and its] therapeutic product should be approved or cleared contemporaneously by FDA for the use indicated in the therapeutic product labeling” [18]. The guidance also stated that, "the results of the IVD companion diagnostic device will be essential for the safe and effective use of the therapeutic product, and its use will be stipulated in the labeling of the therapeutic product". Because the IVD companion diagnostic was identified as essential for this purpose, it was noted that, "with some exceptions FDA does not believe it may approve a novel therapeutic product or new therapeutic product indication for use with an IVD companion diagnostic if the IVD companion diagnostic is not approved or cleared for that indication" [18]. Documents from the FDA and other worldwide regulatory agencies have reiterated the value of companion diagnostics to the personalized medicine process, while also acknowledging the complex development process.

\section{THE DRUG-DIAGNOSTIC CO-DEVELOPMENT PROCESS}

Given the many obstacles to overcome in the pursuit of personalized medicine, leadership at the NIH and the FDA recognized that there was not enough evidence of a down- stream market to entice the private sector to explore most of this scientific potential. In order to counteract this, the agencies began steps to develop a more integrated pathway that connects all the steps between the identification of a potential therapeutic target by academic researchers and the approval of a therapy for clinical use [19].

The FDA's guiding document "Drug-Diagnostic CoDevelopment Concept Paper" was drafted in 2005 to help outline a process to prospectively co-develop a therapeutic product and diagnostic test in a scientifically robust and efficient way [20]. The document identified and outlined the recommended multi-step path from basic research to, ultimately, FDA filing/approval and product launch. Fig. (1) illustrates the many points of contact and feedback required between the traditional pharmaceutical trial process and the parallel development and clinical trials processes for a companion diagnostic.

\section{THE TRADITIONAL DRUG DEVELOPMENT PROCESS}

The traditional drug development and approval process is well established, and outsourcing options are available for pharmaceutical companies. Common partner choices are traditional Contract Research Organizations (CROs) and diagnostics companies. CROs provide efficient, costeffective solutions to conduct clinical research. As well, their focus and expertise is on operational efficiency of clinical trials for pharmaceuticals. This is evidenced by the Association of Clinical Research Organizations (ACRO) Fact Sheet, which states “... In 2008, the average ACRO member company worked on nearly 400 medications in development.”

\section{Integrated Development of a Drug and a Companion Diagnostic}

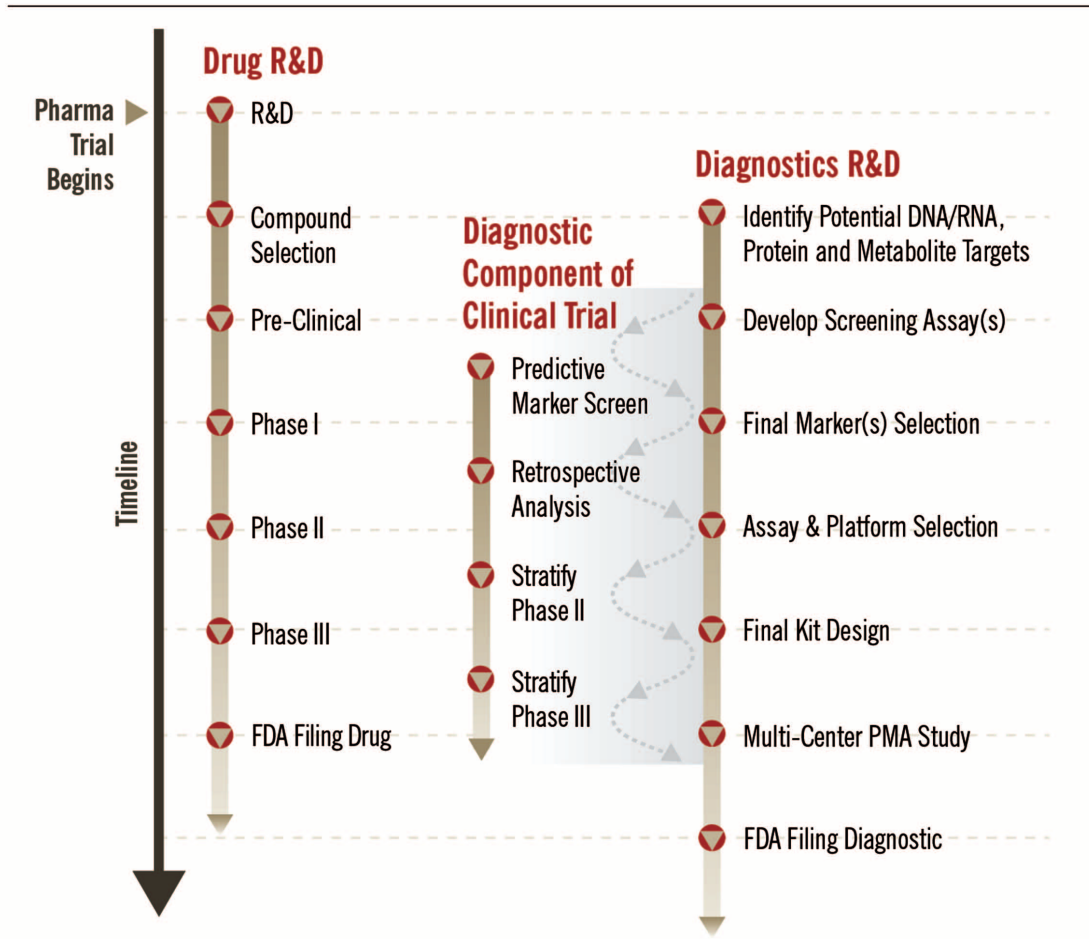

Fig. (1). Key steps in drug-development co-development process [19b]. 
[21]. In contrast, diagnostics companies specialize is the development and manufacturing of diagnostic tests.

\section{THE COMPLEX PATH TO A COMPANION DIAGNOSTIC}

While all regulatory initiatives and guidance documents either directly or indirectly reference companion diagnostic co-development as an important consideration in the new drug approval process, these documents lack clear guidance on how this process should be implemented. The difficulties faced in coordination of therapeutics development with the companion diagnostics process are numerous, and are compounded by the fact that an effective companion diagnostics co-development process does not currently exist, or at best is rudimentary. The typical steps needed for development of a companion diagnostic are outlined in Fig. (2).

Each step of the process requires specialized expertise rarely available in a single organization, thus requiring the coordinated effort of multiple organizations.

\section{TRADITIONAL PARTNERS MAY NOT SUIT COMPANION DIAGNOSTICS}

The need for specific expertise in the diagnostic industry is paramount, as no initial scientific concept for a molecular diagnostic test can be successfully hypothesized or carried out without a strong knowledge base at its core. This begins at the initial assay conceptualization and continues to discovery, optimization, and finally to validation. A considerable hurdle is that few pharmaceutical companies have this expertise in-house, so outsourcing becomes necessary. As a result, many major pharmaceutical companies like Roche, Pfizer, Merck, AstraZeneca, Bristol Myers Squibb, Amgen, Biogen, and Eli Lilly have chosen, or needed, to partner with external companies to develop their own companion diagnostics [22].To date, all of these companies have selected large partners in the diagnostics industry.

In doing so, however, they have potentially created a new problem, in thatone outsourcing partnerchoice - the large diagnostic company - has other motivations, which can complicate the path to a successful companion diagnostic. Diagnostic partners typically have their own product lines and assay platforms, and are therefore predisposed to direct diagnostics development toward an existing technology platform in an effort to capitalize upon internal synergies and increase efficiency, even if that technology may not be the best choice for the new companion diagnostic and therefore, their pharmaceutical partner. The choice of an assay platform also affects deployment into, and acceptance, by the end user clinical laboratories who may offer the test.

A recent example highlights the challenges with pharmadiagnostic company partnerships. Rearrangements of the anaplastic lymphoma kinase $(A L K)$ gene were reported in non-small cell lung cancer (NSCLC) [23]. Subsequently, it was reported that patients with advanced NSCLC containing $A L K$ rearrangements showed a dramatic response to ALKtargeted therapy [23a, 24]. In pretreated patients that generally have a $10 \%$ response rate to conventional chemotherapy, treatment with the oral ALK inhibitor Xalkori ${ }^{\circledR}$ (crizotinib) yielded an overall response rate of 55\% and an estimated sixmonth, progression-free survival rate of 72\% [23a]. Supporting data showed that ALK kinase domain mutations were associated with resistance to treatment, substantiating that ALK was indeed the genetic target of the personalized therapy [23a]. This reinforced that appropriate clinical use of Xalkori ${ }^{\circledR}$ was absolutely dependent upon a companion diagnostic to identify those patients most likely to respond.

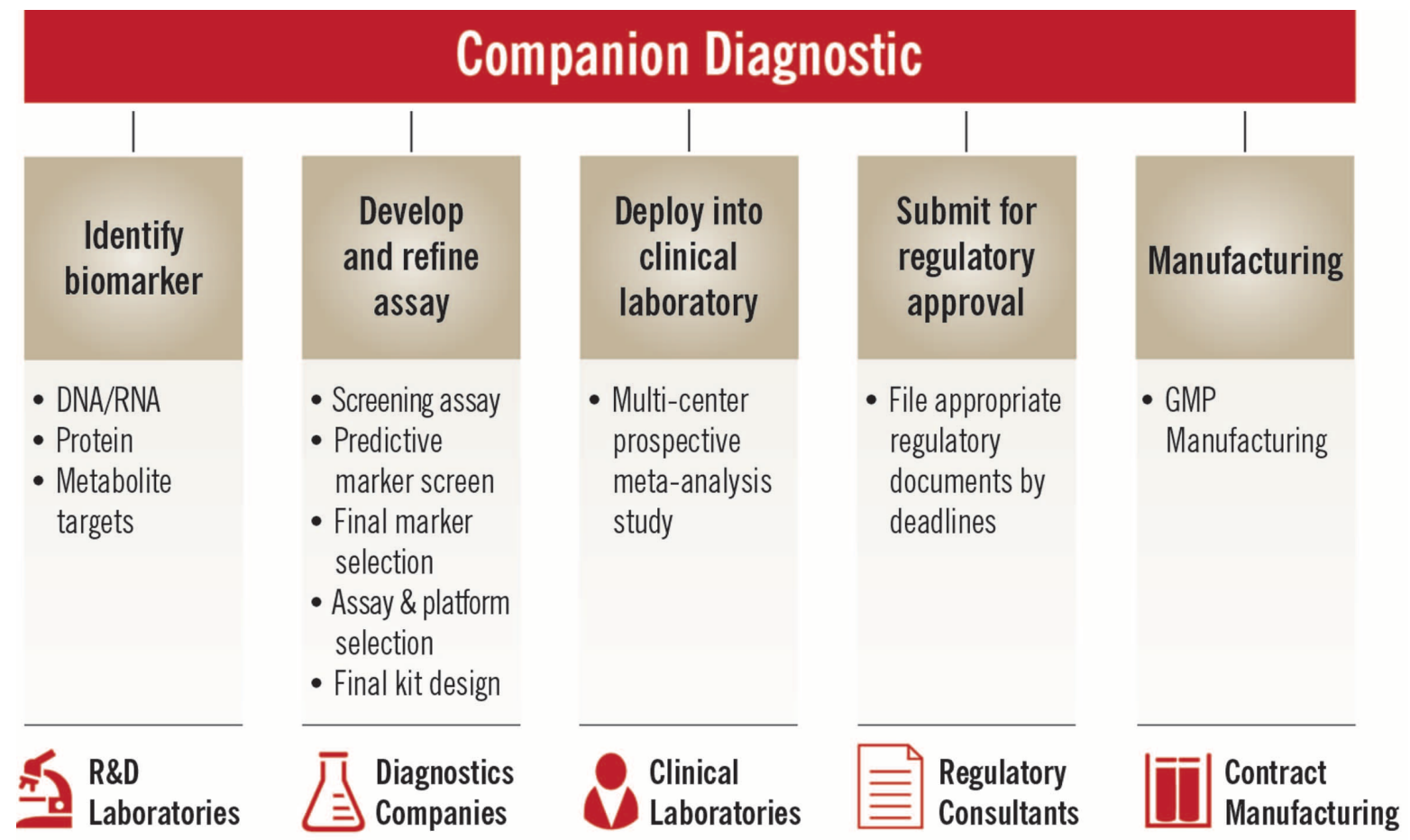

Fig. (2). Typical steps needed to develop a companion diagnostic, separate from drug development. 
As reported in The Wall Street Journal, the codevelopment of a therapeutic and companion diagnostic was problematic from a business perspective [25]. Pfizer developed and manufactures Xalkori. ${ }^{\circledR}$ They partnered with a large diagnostics company, Abbott Molecular, to develop a molecular cytogenetics companion diagnostic test kit for analyzing $A L K$. Early on, Pfizer researchers were reportedly hesitant to supply Abbott with some of its tumor tissue containing $A L K$ gene rearrangements, while expressing concerns that Abbott did not appear to be apprising them on progress developing the test [25]. Abbott also appeared to resist Pfizer's desire to train commercial laboratories on using the test well ahead of FDA approval, because Abbott was concerned that the laboratories could forget how to conduct the assay while waiting for the drug and test to go on sale [25]. Resisting expenditure of resources prior to an FDA approval was potentially another motivation in delaying training. The FDA ultimately approved Xalkori ${ }^{\circledR}$ and its companion diagnostic test kit simultaneously, but not before several delays and difficulties were experienced. The fluorescent in situ hybridization-based assay for $A L K$ rearrangements has the advantage in that it can be performed in many laboratories.

Another partnership choice is the traditional Contract Research Organization (CRO). As described earlier, CROs' expertise is on operational efficiency of clinical trials for pharmaceuticals. This clinical trial experience may be attractive and familiar to pharmaceutical companies looking to outsource this function. However few CROs have experience in IVD development, manufacture, and medical device (companion diagnostic) validation trials. In addition, pharmaceutical companies want to ensure that clinical trials conducted for development of a companion diagnostic are run in an environment that can ensure translation to the clinical setting after marketing launch. Therefore, many are turning to appropriately licensed and accredited clinical laboratories. This necessitates partnering with a laboratory possessing and maintaining those credentials, a service many traditional diagnostics companies and CROs do not offer.

From a diagnostic perspective, co-development of a therapeutic and companion diagnostic is associated with a level of uncertainty. Unlike the pharmaceutical development industry, where there is an understanding that a large number of potential drugs will fail clinical studies (and that the associated revenues will be derived from a very small cohort of successful drugs), no such expectation currently exists in the diagnostic development space. Therefore, while the codevelopment of a diagnostic and therapeutic is advantageous from a cost and efficiency perspective, the high number of late stage compound failures makes a co-development agreement less financially desirable from a diagnostic perspective, due to the significant risk and high cost involved in developing diagnostics for drugs that never receive regulatory approval. Thus, diagnostic companies may be hesitant to share the financial burden for companion diagnostic development.

Lastly, drug developers must overcome several logistical complexities when managing and coordinating multiple partners in the process: Research and Development (initial research and biomarker development), a Diagnostics Company (to develop and possibly manufacture an assay kit), a CRO (to conduct validation trials), regulatory personnel, and Clinical Laboratories (to perform assay validation and trial testing). All of these steps can cause product delay and introduce points of inefficiency (Fig. 3).

Biopharma brings half of the solution

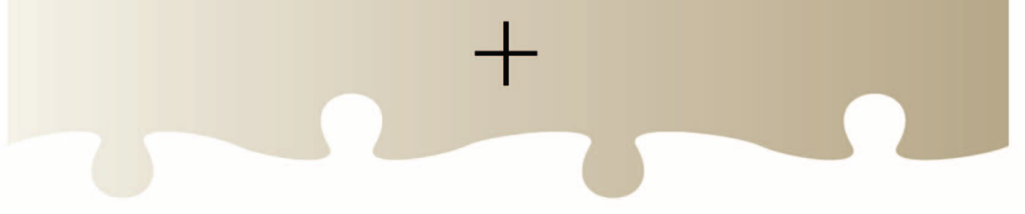
Partners needed for diagnostics knowledge and experience
- Research and lab services
- Regulatory guidance
- Diagnostic development
- Manufacturing

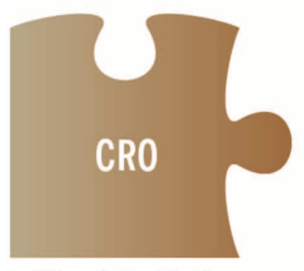

(No clinical lab)

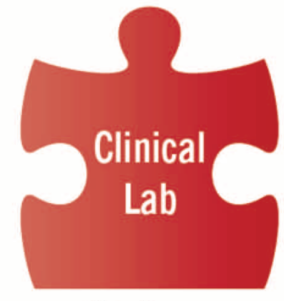

(Don't run clinical trials)

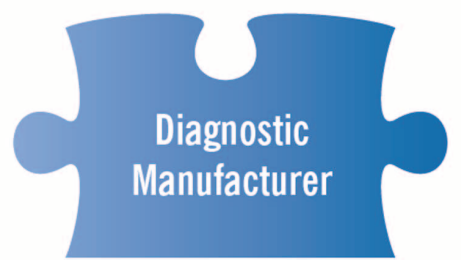

(Incentive to divert development to existing technology platforms)

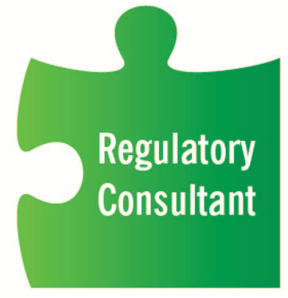

(Not integrated into R\&D)

Fig. (3). Biopharmaceutical companies' traditional role in personalized medicine. 


\section{Biopharma brings half of the solution}

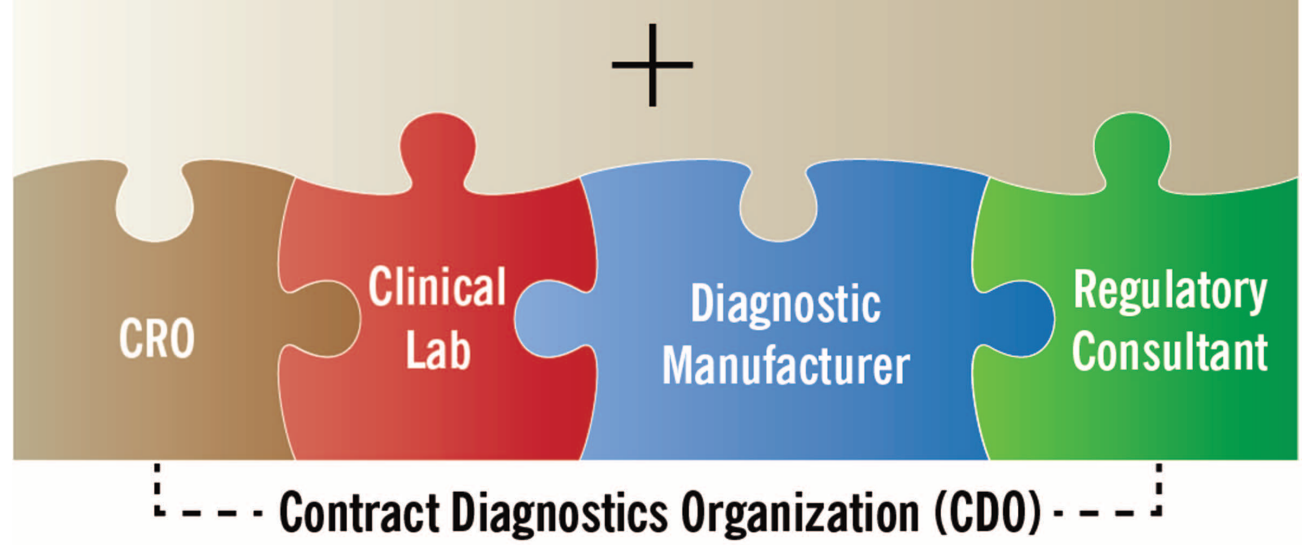

Fig. (4). Various services offered by a CDO, all housed within one organization.

\section{AN INTEGRATED SOLUTION - THE CONTRACT DIAGNOSTICS ORGANIZATION}

The Contract Diagnostics Organization (CDO) is a new business model, which allows pharmaceutical companies to outsource to a single partner offering integrated services within one organization, to initiate the parallel development of companion diagnostic tests in synergy with drug development. The CDO combines diagnostics research, a licensed and accredited clinical laboratory, manufacturing, and consulting, in an integrated, technology-independent manner (Fig. 4).

A CDO can design, manage, and coordinate all aspects of clinical trials for the development of a diagnostic product from assay concept to regulatory submission and commercialization. This can include (but is not limited to) 1) Developing novel assays or validating established assays; 2) Developing, managing, and conducting a clinical research project; 3) Developing a Case Report Form (CRF)/Study document; 4) Submitting and managing Institutional Review Board (IRB) documents; 5) Laboratory services; 6) Managing FDA submissions for $510(\mathrm{k})$ or Premarket Approval (PMA) clearances; and 7) Writing papers for publication.

A CDO partners with clinical laboratories (ideally CLIA certified and CAP accredited), which can offer access to a full complement of specialty testing services.

Additionally, some CDOs have manufacturing capabilities for a specific diagnostic product, which allows them to custom develop IVD kits, reagents, or products for a research project, clinical trial, or product commercialization. Examples include manufacturing reagents, sample collection kits, or entire diagnostic assays.

In addition, a CDO can offer biopharmaceutical and diagnostics consulting services. For example, this can includeconsulting for: 1) Assay development, platform evaluation, and validation; 2) Management of all levels of regulatory compliance, processes, applications, and approvals; 3) FDA interface (product life-cycle support/management); 4) Technology utility, application, fit-for-purpose, and patentability;
5) Market and competitor analyses; 6) Mergers and acquisitions.

In conclusion a CDO eliminates the need for outsourcing to, and coordinating across, multiple partners. It also builds in flexibility and the ability to implement an efficient, nimble strategy that may naturally shift as development continues.

\section{CONCLUSION}

The basic science behind personalized medicine will continue to offer a myriad of choices for pharmaceutical companies to create companion diagnostics in healthcare. The downstream market for patient-customized therapeutics has significant untapped potential. However, the traditional bench-to-bedside development of a pharmaceutical product needs to be coordinated with that of a companion diagnostic. The CDO combines all of the necessary services, including biomarker research, a licensed and accredited clinical laboratory, manufacturing and consulting, in an integrated, technology-independent manner. As a CRO is the standard outsourcing partner for pharmaceutical clinical trials, a Contract Diagnostics Organization is specifically suited for outsourcing of companion diagnostics co-development.

\section{ACKNOWLEDGEMENT}

None Declared.

\section{CONFLICT OF INTEREST}

Drs Cotter and Moore are Principals at ResearchDx, LLC

\section{REFERENCES}

[1] Hoggatt, J. Personalized medicine--trends in molecular diagnostics: exponential growth expected in the next ten years. Mol. Diagn. Ther., 2011, 15 (1), 53-55.

[2] (a) Langreth, R.; Waldholz, M. New era of personalized medicine: targeting drugs for each unique genetic profile. Oncologist, 1999, 4 (5), 426-427;(b) Jorgensen, J. T. New era of personalized medicine: a 10-year anniversary. Oncologist, 2009, 14 (5), 557-558. 
[3] Jorgensen, J. T. A challenging drug development process in the era of personalized medicine. Drug Discov. Today, 2011, 16 (19-20), 891-897.

[4] PWC. The New Science of Personalized Medicine: Translating the promise into practice; Pricewaterhouse Coopers LLP. Report.PWC:UK, October 2009.

[5] Abrahams, E.; Silver, M. The case for personalized medicine. $J$ Diabetes Sci. Technol., 2009, 3 (4), 680-684.

[6] Kongkaew, C.; Noyce, P. R.; Ashcroft, D. M. Hospital admissions associated with adverse drug reactions: a systematic review of prospective observational studies. Ann. Pharmacother., 2008, 42 (7), 1017-1025.

[7] Bates, S. Progress towards personalized medicine. Drug Discov. Today, 2010, 15 (3-4), 115-120.

[8] (a) Piccart-Gebhart, M. J.; Procter, M.; Leyland-Jones, B.; Goldhirsch, A.; Untch, M.; Smith, I.; Gianni, L.; Baselga, J.; Bell, R.; Jackisch, C.; Cameron, D.; Dowsett, M.; Barrios, C. H.; Steger, G.; Huang, C. S.; Andersson, M.; Inbar, M.; Lichinitser, M.; Lang, I.; Nitz, U.; Iwata, H.; Thomssen, C.; Lohrisch, C.; Suter, T. M.; Ruschoff, J.; Suto, T.; Greatorex, V.; Ward, C.; Straehle, C.; McFadden, E.; Dolci, M. S.; Gelber, R. D. Trastuzumab after adjuvant chemotherapy in HER2-positive breast cancer. N. Eng. J. Med., 2005, 353 (16), 1659-1672 (b) Romond, E. H.; Perez, E. A.; Bryant, J.; Suman, V. J.; Geyer, C. E., Jr.; Davidson, N. E.; TanChiu, E.; Martino, S.; Paik, S.; Kaufman, P. A.; Swain, S. M.; Pisansky, T. M.; Fehrenbacher, L.; Kutteh, L. A.; Vogel, V. G.; Visscher, D. W.; Yothers, G.; Jenkins, R. B.; Brown, A. M.; Dakhil, S. R.; Mamounas, E. P.; Lingle, W. L.; Klein, P. M.; Ingle, J. N.; Wolmark, N. Trastuzumab plus adjuvant chemotherapy for operable HER2-positive breast cancer. N. Eng. J. Med., 2005, 353 (16), 1673-1684. (c) Liu, W.; Zhong, S.; Chen, J.; Yu, Y., HER2/neu Overexpression is an independent prognostic factor for intestinal-type and early-stage gastric cancer patients. J. Clin. Gastroenterol., 2012, 46(4), e31-e37. (d) Ruschoff, J.; Hanna, W.; Bilous, M.; Hofmann, M.; Osamura, R. Y.; Penault-Llorca, F.; van de Vijver, M.; Viale, G. HER2 testing in gastric cancer: a practical approach. Mod. Pathol., 2012, 25(5),637-650.

[9] Rettie, A. E.; Korzekwa, K. R.; Kunze, K. L.; Lawrence, R. F.; Eddy, A. C.; Aoyama, T.; Gelboin, H. V.; Gonzalez, F. J.; Trager, W. F. Hydroxylation of warfarin by human cDNA-expressed cytochrome P-450: a role for P-4502C9 in the etiology of (S)warfarin-drug interactions. Chem. Res. Toxicol., 1992, 5 (1), 54-59.

[10] Carlquist, J. F.; Anderson, J. L. Using pharmacogenetics in real time to guide warfarin initiation: a clinician update. Circulation, 2011, 124 (23), 2554-2559.

[11] Ross, D. M.; Hughes, T. P. Current and emerging tests for the laboratory monitoring of chronic myeloid leukaemia and related disorders. Pathology, 2008, 40 (3), 231-246.

[12] Schad, C. R.; Hanson, C. A.; Paietta, E.; Casper, J.; Jalal, S. M.; Dewald, G. W. Efficacy of fluorescence in situ hybridization for detecting PML/RARA gene fusion in treated and untreated acute promyelocytic leukemia. Mayo Clin. Proc., 1994, 69 (11), 10471053.

[13] Ptolemy, A. S.; Rifai, N. What is a biomarker? Research investments and lack of clinical integration necessitate a review of biomarker terminology and validation schema. Scand. J. Clin. Lab. Invest. Suppl., 2010, 242, 6-14.
[14] The Tufts Center for the Study of Drug Development. Lack of clinically useful diagnostics Hinder Growth in Personalized Medicines; Impact Report. Tufts University: USA, Impact Report, 2011, vol. 13, no. 4.

[15] Lievre, A.; Bachet, J. B.; Le Corre, D.; Boige, V.; Landi, B.; Emile, J. F.; Cote, J. F.; Tomasic, G.; Penna, C.; Ducreux, M.; Rougier, P.; Penault-Llorca, F.; Laurent-Puig, P. KRAS mutation status is predictive of response to cetuximab therapy in colorectal cancer. Cancer Res., 2006, 66 (8), 3992-3995.

[16] Papadopoulos, N.; Kinzler, K. W.; Vogelstein, B. The role of companion diagnostics in the development and use of mutationtargeted cancer therapies. Nat. Biotechnol., 2006, 24 (8), 985-995.

[17] PMC. The Case for Personalized Medicine, $3^{\text {rd }}$ ed.; Personalized Medicine Coalition: NY, 2011.

[18] Services, U. S. D. o. H. a. H. Draft Guidance for Industry and Food and Drug Administration Staff - In Vitro Companion Diagnostic Devices. Available at: www.fda.gov/downloads/MedicalDevices/ DeviceRegulationandGuidance/GuidanceDocuments/UCM262327. pdf [Accessed on: $3^{\text {rd }}$ Jan].

[19] (a) Hamburg, M. A.; Collins, F. S. The path to personalized medicine. $N$ Engl J. Med 2010, 363 (4), 301-304 (b) Moore, M. W.; Cotter, P. D. Advancing personalized medicine: outsourcing to a CDO. InsideOutsourcing, 2011, 2011, 36-40.

[20] FDA. Drug-Diagnostic Co-Development Concept Paper (draft concept paper). Department of Health and Human Services (HHS)F, FAD: USA, 2005.

[21] Association of Clinical Research Organization (ACRO). Fact Sheet - Available at: http://www.acrohealth.org/fact-sheet.html [Accessed on: 10th Jan 2012].

[22] Naylor, S.; Cole, T. Overview of companion diagnostics in the pharmaceutic Industry. Drug Discov. World Spring Ed 2010, 2010, 67-79

[23] (a) Gerber, D. E.; Minna, J. D. ALK inhibition for non-small cell lung cancer: from discovery to therapy in record time. Cancer Cell, 2010, 18 (6), 548-551 (b) Soda, M.; Choi, Y. L.; Enomoto, M.; Takada, S.; Yamashita, Y.; Ishikawa, S.; Fujiwara, S.; Watanabe, H.; Kurashina, K.; Hatanaka, H.; Bando, M.; Ohno, S.; Ishikawa, Y.; Aburatani, H.; Niki, T.; Sohara, Y.; Sugiyama, Y.; Mano, H. Identification of the transforming EML4-ALK fusion gene in nonsmall-cell lung cancer. Nature, 2007, 448 (7153), 561-566.

[24] (a) Choi, Y. L.; Soda, M.; Yamashita, Y.; Ueno, T.; Takashima, J.; Nakajima, T.; Yatabe, Y.; Takeuchi, K.; Hamada, T.; Haruta, H.; Ishikawa, Y.; Kimura, H.; Mitsudomi, T.; Tanio, Y.; Mano, H., EML4-ALK mutations in lung cancer that confer resistance to ALK inhibitors. N Engl J. Med., 2010, 363 (18), 1734-1739 (b) Kwak, E. L.; Bang, Y. J.; Camidge, D. R.; Shaw, A. T.; Solomon, B.; Maki, R. G.; Ou, S. H.; Dezube, B. J.; Janne, P. A.; Costa, D. B.; Varella-Garcia, M.; Kim, W. H.; Lynch, T. J.; Fidias, P.; Stubbs, H.; Engelman, J. A.; Sequist, L. V.; Tan, W.; Gandhi, L.; Mino-Kenudson, M.; Wei, G. C.; Shreeve, S. M.; Ratain, M. J.; Settleman, J.; Christensen, J. G.; Haber, D. A.; Wilner, K.; Salgia, R.; Shapiro, G. I.; Clark, J. W.; Iafrate, A. J. Anaplastic lymphoma kinase inhibition in non-small-cell lung cancer. $N$ Engl J. Med., 2010, 363 (18), 1693-1703.

[25] Rockoff, J. D. Tests are key but partners tricky. Wall Street J., 2011. [ E-pub ahead of print]

(c) Cotter et al.; Licensee Bentham Open.

This is an open access article licensed under the terms of the Creative Commons Attribution Non-Commercial License (http://creativecommons.org/licenses/by-nc/3.0/) which permits unrestricted, non-commercial use, distribution and reproduction in any medium, provided the work is properly cited.. 